\title{
Decrease in antioxidant status of plasma and erythrocytes from geriatric population
}

\author{
Manjulata Kumawat ${ }^{\mathrm{a}, 1}$, Tarun Kumar Sharma ${ }^{\mathrm{a}, 1, *}$, Ishwar Singh ${ }^{\mathrm{b}, 1}$, Neelima Singh ${ }^{\mathrm{c}, 1}$, \\ Sanjeev Kumar Singh ${ }^{\mathrm{c}, 1}$, Veena Singh Ghalaut ${ }^{\mathrm{a}, 1}$, Vijay Shankar ${ }^{\mathrm{a}}$ and Satish Kumar Vardey ${ }^{\mathrm{d}, 1}$ \\ ${ }^{a}$ Department of Biochemistry, Pt. B.D. Sharma University of Health Sciences, PGIMS, Rohtak, Haryana, India \\ ${ }^{\mathrm{b}}$ Department of Neurosurgery, Pt. B.D. Sharma University of Health Sciences, PGIMS, Rohtak, Haryana, India \\ ${ }^{\mathrm{c}}$ Department of Biochemistry, G.R. Medical College, Gwalior, Madhya Pradesh, India \\ ${ }^{\mathrm{d}}$ Department of Biochemistry, S.M.S. Medical College, Jaipur (Rajasthan), India
}

\begin{abstract}
Background: Ageing is associated with an accumulation of free radical damage, which leads to physiological and clinical modifications. The study aims to find out the status of lipid profile, antioxidant enzymes, malondialdehyde in geriatric population.

Patients/methods: The study was conducted on 150 subjects (75 healthy control between the ages of 20-30 years and 75 elderly subjects between ages of 50-70 years as cases). The following parameters were analyzed using the standard reference methods: lipid profile, reduced glutathione, glutathione peroxidase, glutathione reductase, catalase, superoxide dismutase and malondialdehyde.

Results: The present study was conducted to estimate the oxidative stress parameters in geriatric population. Highly significant increase in total cholesterol (TC), triglyceride (TG), LDL-cholesterol (LDL-C), VLDL-cholesterol (VLDL-C), malondialdehyde, catalase and decrease in high density lipoprotein cholesterol (HDL-C), reduced glutathione, glutathione peroxidase, glutathione reductase, superoxide dismutase was observed in geriatrics when compared with their younger counterparts.

Conclusion: This study concluded that there is enhanced oxidative stress and decreased antioxidant defence in geriatrics as compared to younger subjects which could play an important role in ageing. Dyslipidemia has become one of the important risk factors for the increasing prevalence of cardiovascular diseases. There is lack of awareness on the relationship between blood lipids and the risk of cardiovascular diseases in geriatric population. The strategy of early prevention should be adopted against dyslipidemia.
\end{abstract}

Keywords: Oxidative stress, malondialdehyde, antioxidants, geriatric population

List of abbreviations

CAT Catalase;

MDA Malondialdehyde;

GSH Reduced glutathione;

GR Glutathione reductase;

GPx Glutathione peroxidase;

$\begin{array}{ll}\text { SOD } & \text { Superoxide dismutase; } \\ \text { TC } & \text { Total cholesterol; } \\ \text { TG } & \text { Triglyceride; } \\ \text { HDL-C } & \text { HDL-Cholesterol; } \\ \text { LDL-C } & \text { LDL-Cholesterol; } \\ \text { VLDL-C } & \text { VLDL-Cholesterol }\end{array}$

\section{Introduction}

${ }^{1}$ These authors contributed equally to the skillful editing of the manuscript, interpretation of results and statistical analysis.

${ }^{*}$ Corresponding author: Tarun Kumar Sharma, Department of Biochemistry, Pt. B.D. Sharma, University of Health Sciences, P.G.I.M.S., Rohtak (Haryana), 124001, India. Tel.: +91 989697 6598, +91 9468599091; E-mail: Sharma_bio82@yahoo.co.in.
Damage caused by oxygen radicals is responsible for many of the changes associated with ageing. Ageing is a progressive accumulation of physiological and morphological changes, responsible for an increasing 
susceptibility to disease [1]. Oxygen free radicals are implicated in the ageing process. The univalent reduction of oxygen results in a series of cytotoxic oxygen species. These highly reactive species can cause cell damage including lipid peroxidation, inactivation of enzymes, alteration of intra-cellular oxidation-reduction state and damage to DNA [2-4]. Ageing is a process of irreversible changes associated with accumulation of these oxidative damages in the cell. However free radicals do not go unchecked. Mounted against them is a multilayer defense system manned by antioxidants that react with and disarm these damaging molecules. The human body has a complex antioxidant defense system that includes enzymes like superoxide dismutase (SOD), glutathione peroxidase (GPx), glutathione reductase (GR), catalase (CAT) and non-enzymes like glutathione (GSH) [5,6]. Since age related changes in antioxidants in erythrocytes have not been systematically studied, the objective of the present study was to evaluate the level of malondialdehyde (MDA) and antioxidants SOD, GPx, GR, CAT and G-SH in elderly people.

Blood lipid is an important indicator for health. In this study, total cholesterol, triglyceride (TG), LDLcholesterol, VLDL-cholesterol were significantly higher while HDL-cholesterol was significantly lower in geriatric population when compared with young subjects.

Thus, screening for dyslipidemia in the elderly is recommended. On the other hand, there is no strong evidence that cholesterol lowering drugs are cost-effective in primary prevention of coronary heart disease in this group of people. So, the National Cholesterol Education Program (NCEP III, 2001) has recommended diet therapy and lifestyle modification as major strategies in lowering cholesterol level in primary prevention of coronary artery disease and limited use of cholesterol lowering drugs to only in high risk elderly who are otherwise in good health [7]. Although, a large number of epidemiological and clinical studies have analyzed the potential therapeutic utility of antioxidants [8], longterm epidemiological data showing the association of oxidative stress and mortality in humans is lacking [9]. Still, we do not know the actual importance of oxidative mechanisms such as lipoperoxidation in predicting the chance of death, either.

\section{Materials and methods}

The study involved 150 subjects which consisted of 75 (40 males, 35 females) healthy people between the ages of $20-30$ years as controls and 75 (40 males, 35 females) elderly subjects between the ages of 5070 years as cases. Elderly people taking any drugs or any antioxidant supplementation were excluded from the study to avoid the possible confounding effect of such factors on lipid peroxidation and antioxidant enzymes. Subjects with diabetes, hypertension, stroke, renal failure, myocardial infarction, acute and chronic inflammatory conditions were excluded from the study. Chronic smokers, chronic alcoholics and the patients with symptomatical diseases of coronary artery disease and peripheral artery disease (atherosclerosis) were also excluded from the study. Written informed consent of all subjects was taken before initiating the study. All ethical measures were taken prior to and during the study.

\subsection{Collection of blood samples}

Under aseptic conditions, $4 \mathrm{~mL}$ of venous blood was collected from each subject. This was distributed in following vials:

- $0.4 \mathrm{~mL}$ in heparinized vial for estimation of reduced glutathione (GSH)

- $1.6 \mathrm{~mL}$ in citrated vial for estimation of catalase (CAT), glutathione reductase (GR), glutathione peroxidase (GPx) and malondialdehyde (MDA).

$-2 \mathrm{~mL}$ in plain vial for estimation of superoxide dismutase (SOD) and lipid profile in serum.

\subsection{Preparation of haemolysate}

$2.0 \mathrm{~mL}$ blood containing citrate was centrifuged at $2000 \mathrm{rpm}$ for 10 minutes. Plasma separated out for estimating MDA. The packed cell volume (PCV) was washed two times with normal saline. After that PCV was mixed gently and $1 \mathrm{~mL}$ of DW was added to it. Mixture was refrigerated for 10 minutes and then vigorously shaken for 12 minutes for lysis of the cell. To this $0.5 \mathrm{~mL}$ of chloroform was added as a preservative. Mixture was centrifuged at $3000 \mathrm{rpm}$ for 20 minutes. Mixture was clearly separated into 3 layers, lower most layer of chloroform, middle of cell stroma and upper most layer of hemolysate, which was pipetted off carefully and filtered through two layers of filter paper into clear dry test tube. In this hemolysate, glutathione peroxidase, glutathione reductase and catalase were estimated.

The blood samples were analyzed spectrophotometrically for the following parameters using the standard reference methods: serum triglycerides [10], serum 
Table 1

Showing the status of lipid profile, GSH, antioxidant enzymes and malondialdehyde (MDA) in geriatric and control group

\begin{tabular}{|c|c|c|c|}
\hline Parameters & Control group $n=75$ Median (Range) & Geriatric group $n=75$ Median (Range) & $\mathrm{p}$ value \\
\hline $\mathrm{TC}(\mathrm{mmol} / \mathrm{L})$ & $4.81(4.2-5.98)$ & $5.71(4.21-6.33)$ & $p<0.05$ \\
\hline $\mathrm{TG}(\mathrm{mmol} / \mathrm{L})$ & $1.29(0.99-1.61)$ & $1.36(0.99-1.61)$ & $p<0.05$ \\
\hline HDL-C (mmol/L) & $1.24(0.79-1.95)$ & $1.14(0.79-1.95)$ & $p<0.05$ \\
\hline LDL-C (mmol/L) & $3.1(1.83-4.16)$ & $3.65(2.28-4.67)$ & $p<0.05$ \\
\hline VLDL-C (mmol/L) & $0.59(0.45-0.74)$ & $0.62(0.45-0.74)$ & $p<0.05$ \\
\hline $\mathrm{GSH}(\mathrm{mg} \%)$ & $25.23(12.39$ to 35.12$)$ & $14.3(10.0$ to 18.0$)$ & $p<0.001$ \\
\hline GPx (Units/gmHb) & $10.6(8.0$ to 16.0$)$ & $8.3(6.2$ to 11.0$)$ & $p<0.001$ \\
\hline GR (Units/gmProtein) & 18.7 (18.0 to 20.8$)$ & $16.6(10.1$ to 18.3$)$ & $p<0.001$ \\
\hline CAT (Units/gmProtein/mL) & $5.3(4.0$ to 7.0$)$ & $5.8(4.9$ to 7.0$)$ & $p<0.001$ \\
\hline SOD Units/mgProtein/mL) & $8.4(6.1$ to 10.7$)$ & $6.5(4.3$ to 8.9$)$ & $p<0.001$ \\
\hline MDA (nmol/mL) & $3.3(2.0$ to 6.0$)$ & $5.1(3.0$ to 6.0$)$ & $p<0.001$ \\
\hline
\end{tabular}

total cholesterol [11], serum HDL-cholesterol [12]. Serum LDL-cholesterol and serum VLDL-cholesterol were calculated by the Friedewald formula [13]. Reduced glutathione [14], glutathione peroxidase [15], glutathione reductase [16], catalase [17] were estimated in erythrocytes. Superoxide dismutese was estimated in serum [18]. Malondialdehyde was estimaetd in plasma [19]. The samples were stored at $+2^{\circ} \mathrm{C}$ to $+8^{\circ} \mathrm{C}\left(+4^{\circ} \mathrm{C}\right)$ before analysis and all the samples were analysed on the same day of collection.

Reduced Glutathione (GSH) level was determined according to Beutler et al. [14] in a meta-phosphoric acid filtrate by using 5,5' -dithiobis-2-nitrobenzoic acid (DTNB). Enzymatic activity was expressed in $\mathrm{mg} \%$. $\mathrm{Mg} \%$ of glutathione in blood in three coleman junior instruments identical $\mathrm{E}$ values were found that is 0.542 .

Glutathione peroxidase (GPx) activity was determined according to Hafeman et al. [15] by using hydrogen peroxide as a substrate. Enzymatic activity was expressed in $\mathrm{U} / \mathrm{gmHb}$.

Glutathione reductase (GR) was determined according to Horn et al. [16] which catalyses the reduction of GSSG in the presence of NADPH which is oxidized to NADP ${ }^{+}$. Enzymatic activity was expressed in Units/gm Protein.

Catalase (CAT) was estimated by the method of Sinha [17] and was assayed colorimetrically at $620 \mathrm{~nm}$. The method is based on the fact that dichromate in acetic acid is reduced to chromic acetate when heated in the presence of hydrogen peroxide $\left(\mathrm{H}_{2} \mathrm{O}_{2}\right)$, with the formation of perchromic acid as an unstable intermediate. The chromic acetate thus produced is measured colorimetrically. Catalytic activity was expressed in Units/gm Protein/mL.

The activity of superoxide dismutase (SOD) was assayed by applying the method of Mishra et al. [18]. The ability of superoxide dismutase to inhibit the autooxidation of epinephrine at $\mathrm{pH} 10.2$ has been used as the basis of a convenient and sensitive assay for this enzyme. Enzymatic activity was expressed in $\mathrm{U} / \mathrm{mg}$ Protein/mL.

Plasma malondialdehyde (MDA) was estimated by method of Jean et al. [19]. After the reaction of thiobarbituric acid with malondialdehyde, the reaction product was extracted in butanol and was measured. Enzymatic activity was expressed in $\mathrm{nmol} / \mathrm{mL}$.

\subsection{Statistical analysis}

All results were expressed in Median (Range). Differences between the Median (Range) were calculated by Mann-Whitney U test. The statistical analysis was performed using SPSS version 17.0. Pearson correlation coefficients were used to analyze relationships between biochemical parameters. The level of significance was set as $\mathrm{p}$ value $<0.001$ to be significant and $\mathrm{p}$ value $>0.05$ as non-significant.

\section{Results}

All results were expressed in Median (Range) (Table 1). In our study we observed a significant decrease $(p<0.001)$ in reduced glutathione $(\mathrm{GSH})$, glutathione peroxidase (GPx), glutathione reductase (GR), and superoxide dismutase (SOD) in geriatric population as compared to the control group. However there is a significant increase $(p<0.001)$ in the level of catalases (CAT) and malondialdehyde (MDA).

We also observed a significant decrease $(p<0.05)$ in (HDL- C) high density lipoprotein cholesterol and there is a significant increase $(p<0.05)$ in total cholesterol (TC), triglyceride (TG), LDL-cholesterol (LDLC), VLDL-cholesterol (VLDL-C).

The Pearson correlations data between all the study parameters with lipid profile, GSH, antioxidant en- 
Table 2

Showing the Pearson correlations data between all the study parameters with lipid profile, GSH, antioxidant enzymes and MDA in geriatric population

\begin{tabular}{|c|c|c|c|c|c|c|c|c|c|c|}
\hline Parameters & TG & HDL-C & LDL-C & VLDL-C & GSH & GPx & GR & CAT & SOD & MDA \\
\hline \multirow[t]{2}{*}{$\mathrm{TC}$} & $r=0.49$ & $r=-0.32$ & $r=0.97$ & $r=0.61$ & $r=-0.39$ & $r=-0.30$ & $r=-0.48$ & $r=0.24$ & $r=-0.42$ & $r=0.42$ \\
\hline & $p<0.05$ & $p<0.05$ & $p<0.05$ & $p<0.05$ & $p<0.05$ & $p<0.05$ & $p<0.05$ & $p<0.05$ & $p<0.05$ & $p<0.05$ \\
\hline \multirow[t]{2}{*}{ TG } & - & $r=-0.33$ & $r=0.39$ & $r=0.72$ & $r=-0.56$ & $r=-0.45$ & $r=-0.56$ & $r=0.23$ & $r=-0.37$ & $r=0.55$ \\
\hline & & $p<0.05$ & $p<0.05$ & $p<0.05$ & $p<0.05$ & $p<0.05$ & $p<0.05$ & $p<0.05$ & $p<0.05$ & $p<0.05$ \\
\hline \multirow[t]{2}{*}{ HDL-C } & - & - & $r=-0.39$ & $r=-0.43$ & $r=0.42$ & $r=0.33$ & $r=0.40$ & $r=-0.24$ & $r=0.26$ & $r=-0.30$ \\
\hline & & & $p<0.05$ & $p<0.05$ & $p<0.05$ & $p<0.05$ & $p<0.05$ & $p<0.05$ & $p<0.05$ & $p<0.05$ \\
\hline \multirow[t]{2}{*}{ LDL-C } & - & - & - & $r=0.46$ & $r=-0.35$ & $r=-0.27$ & $r=-0.41$ & $r=0.22$ & $r=-0.40$ & $r=0.36$ \\
\hline & & & & $p<0.05$ & $p<0.05$ & $p<0.05$ & $p<0.05$ & $p<0.05$ & $p<0.05$ & $p<0.05$ \\
\hline \multirow[t]{2}{*}{ VLDL-C } & - & - & - & - & $r=-0.56$ & $r=-0.41$ & $r=-0.64$ & $r=0.31$ & $r=-0.37$ & $r=0.52$ \\
\hline & & & & & $p<0.05$ & $p<0.05$ & $p<0.05$ & $p<0.05$ & $p<0.05$ & $p<0.05$ \\
\hline \multirow[t]{2}{*}{ GSH } & - & - & - & - & - & $r=0.54$ & $r=0.57$ & $r=-0.094$ & $r=0.54$ & $r=-0.044$ \\
\hline & & & & & & $p<0.05$ & $p<0.05$ & $p>0.05$ & $p<0.05$ & $p>0.05$ \\
\hline \multirow[t]{2}{*}{ GPx } & - & - & - & - & - & - & $r=0.62$ & $r=-0.183$ & $r=0.52$ & $r=0.121$ \\
\hline & & & & & & & $p<0.05$ & $p>0.05$ & $p<0.05$ & $p>0.05$ \\
\hline \multirow[t]{2}{*}{ GR } & - & - & - & - & - & - & - & $r=-0.182$ & $r=0.46$ & $r=-0.023$ \\
\hline & & & & & & & & $p>0.05$ & $p<0.05$ & $p>0.05$ \\
\hline \multirow[t]{2}{*}{ CAT } & - & - & - & - & - & - & - & - & $r=0.155$ & $r=-0.162$ \\
\hline & & & & & & & & & $p>0.05$ & $p>0.05$ \\
\hline SOD & - & - & - & - & - & - & - & - & - & $\begin{array}{l}r=-0.037 \\
p>0.05\end{array}$ \\
\hline
\end{tabular}

Correlation is significant at 0.05 levels (2- tailed); $p>0.05$, Non-significant; $p<0.05$, Significant.

zymes and MDA in geriatric population is shown in Table 2.

The significant negative correlation were observed in HDL-C, GSH, GPx, GR and SOD with TC and TG; LDL-C, VLDL-C, CAT and MDA with HDL-C; GPx, GR and SOD with LDL-C and the significant positive correlation in LDL-C, VLDL-C, CAT and MDA with TC and TG; GSH, GPx, GR and SOD with HDL-C; VLDL-C, CAT and MDA with LDL-C in geriatric population $(p<0.05$, Table 2$)$.

The significant positive correlation were observed in GSH with GPx, GR and SOD; GPx with GR and SOD; GR with SOD $(p<0.05$, Table 2$)$.

The non-significant negative correlation were observed in CAT with GSH, GPx, GR and MDA and positive correlation with SOD. Also non-significant negative correlation were observed in MDA with GSH, GR and SOD and positive correlation with GPx in geriatric population ( $p>0.05$, Table 2$)$.

\section{Discussion}

The oxidative stress theory of aging offers the best mechanistic elucidation of the aging phenomenon and other age-related diseases. Susceptibility of the oxidative damage mechanisms operating in the body which are induced to maintain the antioxidant capacity during aging [20]. The evidences of potential beneficial role of exogenous antioxidants in preventing atherosclerot- ic disease are ambiguous. Although experimental evidences strongly suggest that oxidized LDL accelerates atherosclerosis, the epidemiologic evidences from clinical trials are not entirely consistent with the potential role of exogenous antioxidants in preventing atherosclerotic disease [21].

Aging is associated with changes in physical characteristics and decline of many physiological functions. The excessive production of free radicals in the organism, and the imbalance between the concentrations of these and the antioxidant defenses may be related to aging [22,23]. Oxidative stress results from the imbalance between oxidative and antioxidative mechanisms with increased levels of pro-oxidants and depletion of antioxidants leading to tissue damage. MDA is a three carbon, low molecular weight aldehyde that can be produced from free radical attack on polyunsaturated fatty acids of biological membranes. In the present study, we found serum MDA levels were significantly higher in the group of elderly persons, as compared with the younger group.

Lipid peroxidation occurs as a result of oxidative destruction of polyunsaturated fatty acids (PUFAs) located in cell membranes that are readily attacked by oxidizing radicals [24]. The lipid peroxidation proceeds as self-perpetuating chain reaction to form lipid peroxides and aldehydes like MDA which are cytotoxic and mutagenic [25]. Moreover, lipid peroxidation has been implicated in a wide range of tissue injuries and diseases e.g. atherosclerosis and coronary artery disease. 
The present study shows that levels of GSH, SOD, GPx and GR were significantly decreased and that of CAT was increased. The antioxidant enzyme superoxide dismutase (SOD), the most important enzyme present virtually in all aerobic organisms, catalyzes the dismutation of superoxide into oxygen and hydrogen peroxide. Glutathione peroxidase is a selenoenzyme which catalyzes the reduction of hydroperoxides at the expense of reduced glutathione. Catalase is a primary antioxidant defense component that works to catalyze the decomposition of hydrogen peroxide to water, sharing this function with GPx [26].

The hypothesis that the antioxidative capacity is lowered in older subjects is supported by our data for SOD. This is in line with findings of other studies indicating that older subjects had lowered SOD activities [27,28]. A decrease of SOD with aging might be due to a lowered metabolic rate and oxygen consumption generating less ROS, and thereby a lower demand for SOD.

SOD and GPx concentrations are reduced as they continuously work to neutralize the superoxides, hydrogen peroxide and hydroperoxides produced in increased amount after menopause. Levels of CAT were observed to be increased which may be explained by the fact that the activity of catalase increases when the level of GPx decreases because they both work together to perform similar task of neutralizing hydrogen peroxide [29]. Proper antioxidants plus an appropriate diet, have health benefit to reduce morbidity and perhaps increase the healthy, useful life span of an individual [30].

In this study, total cholesterol, triglyceride (TG), LDL-cholesterol, VLDL-cholesterol were significantly higher while HDL-cholesterol was significantly lower in geriatric population when compared with young subjects. It has been concluded that in aging men low serum testosterone concentration is associated with a potentially atherogenic lipid profile, characterized by low HDL cholesterol and high triglycerides concentrations [31].

A number of trials have indeed shown a clear association between dyslipidemia and intima-media thickness (IMT) $[32,33]$. The ageing process by itself causes changes both in the architecture and constitution of the vascular wall. The aging endothelium releases less nitric oxide (NO), which is important for vascular relaxation. The collagen increases, whereas elastin becomes progressively disrupted, thinner, and often fragments itself. Deposits of fat and calcium are formed, with concurrent loss of elasticity. These aging-related vascular changes have important medical implications in CVD pathogenesis [34]. On the other hand, evi- dence that hyperlipemia leads to carotid IMT [35] has triggered a number of studies with ultrasound assessment, which have shown a positive relationship between carotid IMT and LDL-C, even in elderly subjects. In regards to dyslipidemia, observation studies in elderly populations have showed contradictory results. O'Leary and colleagues (1996) investigated a population of 5117 subjects with 65 years or older, and found a positive relation between high levels of LDL-c and IMT, while high levels of HDL-c presented a negative relationship [36]. Bonithon-Kopp and associates (1996) observed a positive relationship between cholesterol levels and presence of plaques in the carotid arteries; however, no relationship was found for IMT [35].

\section{Conclusion}

It is evident from this study that there is enhanced oxidative stress and decreased antioxidant defence in elderly group as compared to young subjects which can play an important role in ageing. In conclusion, the antioxidant defense mechanisms are not sufficient to prevent age related increase in oxidative damage and additional dietary intake of a variety of antioxidants might be beneficial for preserving the normal function in elderly people. Therefore antioxidants in the form of micronutrients and vitamins may be given as supplements in elderly people.

Future studies will need to address targeting therapy to specific tissues and finding appropriate markers for functional consequences of the attenuation of oxidative stress in aging.

As patients grow older, they are more likely to have multiple chronic diseases, including CVD, cerebrovascular disease, kidney disease, diabetes, and metabolic syndrome. Older patients also are more likely to have decreased hepatic function and decreased muscle mass. These factors can all influence screening and treatment decisions in elderly patients.

\section{Acknowledgements}

We are indebted to the staff of the Department of Biochemistry, G.R. Medical College, Gwalior (MP) for their technical assistance. All authors contributed to the skilful editing of the manuscript and interpretation of results. No financial aid or any other type of grant was accepted for this work. 


\section{Conflict of interest}

The authors declare that they have no competing interests.

\section{References}

[1] L.S. Matsubara, P.E. Machado, Age-related changes of glutathione content, glutathione reductase and glutathione peroxidase activity of human erythrocytes. Braz J Med Biol Res 24 (1991), 449-54.

[2] I. Ceballos-Picot, J.M. Trivier, A. Nicole, P.M. Sinet, M. Thevenin, Age-correlated modifications of copper-zinc superoxide dismutase and glutathione-related enzyme activities in human erythrocytes. Clin Chem 38 (1992), 66-70.

[3] D. Harman, Free radicals in aging. Mol Cell Biochem 84 (1988), 155-61.

[4] B. Halliwell, J.M. Gutteridge, Oxygen toxicity, oxygen radicals, transition metals and disease. Biochem J 219 (1984), $1-14$.

[5] W.F. Ward, W. Qi, H. Van Remmen, W.E. Zackert, L.J. Roberts, A. Richardson, Effects of age and caloric restriction on lipid peroxidation: measurement of oxidative stress by F2isoprostane levels. J Gerontol A Biol Sci Med Sci 60 (2005), 847-51.

[6] B. D'Souza, V. D'Souza, Oxidative injury and antioxidant vitamin $\mathrm{E}$ and $\mathrm{C}$ in schizophrenia. Ind J Clin Biochem 18 (2002), 87-90.

[7] Executive Summary of The Third Report of The National Cholesterol Education Program (NCEP 2001) Expert Panel on Detection, Evaluation, And Treatment of High Blood Cholesterol In Adults (Adult Treatment Panel III). JAMA 285(19): 2486-97.

[8] MRC/BHF Heart protection study of antioxidant vitamin supplementation in 20,536 high-risk individuals: A randomized placebo-controlled trial. Lancet 360 (2002), 23-33.

[9] T.R. Golden, A. Hinerfeld, S. Melov, Oxidative stress and aging: Beyond correlation. Aging Cell 1 (2002), 117-23.

[10] G. Buccolo, H. David, Quantitative determination of serum triglycerides by the use of enzymes. Clin Chem 19 (1973), 476-82.

[11] C.C. Allain, L.S. Poon, C.S.G. Chan, W. Richmond, P.C. Fu, Enzymatic determination of total serum cholesterol. Clin Chem 20 (1974), 470-75.

[12] G. Assman, H. Schriewer, G. Schmitz, E.O. Hagele, Quantification of high density lipoprotein cholesterol by precipitation with phosphotungstic acid/MgCl2. Clin Chem 29 (1983), 2026-30.

[13] W.T. Friedwald, R.I. Levy, D.S. Fredrickson, Estimation of concentration of Low Density Lipoprotein Cholesterol in plasma, with-out use of preparative ultracentrifuge. Clin Chem 18 (1972), 499-502.

[14] E. Beutler, O. Duron, B.M. Kelly, Improved method for the determination of blood glutathione. J Lab Clin Med 61(1963), 882-8.

[15] D.G. Hafeman, R.A. Sunde, W.G. Hoekstra, Effect of dietary selenium on erythrocyte and liver glutathione peroxidase in the rat. Nature 104 (1971), 580-7.

[16] H.D. Horn, Glutathione Reductase. In: Bergmayer HU ed. Method in enzymatic analysis New York: Academic press (1963), p875-9.
[17] K.A. Sinha, Colorimetric assay of catalase. Anal Biochem 47 (1972), 389-94.

[18] H.P. Mishra, I. Fridovich, The role of superoxide anion in the auto-oxidation of epinephrine and simple assay for superoxide dismutase. J Biol Chem 247 (1972), 3170-5.

[19] C.D. Jean, T. Maryse, J.F. Marie, Plasma malondialdehyde levels during myocardial infarction. Clin Chim Acta 129 (1983), 319-22.

[20] S.I. Rizvi, P.K. Maurya, Alterations in antioxidant enzymes during aging in humans. Mol Biotechnol 37 (2007), 58-61.

[21] F.J. Nieto, C. Iribarren, M.D. Gross, G.W. Comstock, R.G. Cutler, Uric acid and serum antioxidant capacity: A reaction to atherosclerosis? Atherosclerosis 148 (2000), 131-39.

[22] M.E. Inal, G. Kaubak, E. Sunal, Antioxidant enzyme activities and malondialdehyde levels related to aging. Clin Chim Acta 305 (2001), 75-80.

[23] I.B. Afanas'ev, Free radical mechanism of aging processes under physiological conditions. Biogerontol 6 (2005), 283-90.

[24] B.A. Svingen, J.A. Buege, F.O. O'Neal, S.D. Aust, The mechanism of NADPH-dependent lipid peroxidation. J Biol Chem 254 (1979), 5892-99.

[25] L.J. Marnett, Lipid peroxidation-DNA damage by malondialdehyde. Mutation res 424 (1999), 83-95.

[26] I. Cotgreave, P. Moldeus, S. Orrenius, Host biochemical defense mechanisms against prooxidants. Annu Rev Pharmacol Toxicol 28 (1988), 189-212.

[27] M.E. Inal, G. Kanbak, E. Sunal, Antioxidant enzyme activities and malondialdehyde levels related to aging. Clin Chim Acta 305 (2001), 75-80.

[28] O. Pansarasa, L. Castagna, B. Colombi, J. Vecchiet, G. Felzani, F. Marzatico, Age and sex differences in human skeletal muscle: Role of reactive oxygen species. Free Radic Res 33 (2000), 287-93.

[29] B. Olivier, E.G. Amanda, L. Jianrong, W. Hong, J.V. Joseph, A.R. Paul, Glutathione Peroxidase-Catalase cooperativity is required for resistance to hydrogen peroxide by mature rat oligodendrocytes. J Neuroscience 24 (2004), 1531-40.

[30] D.R. Suresh, K. Sendil, V. Annam, Age related changes in Malondialdehyde: Total Antioxidant Capacity Ratio - a novel marker of oxidative stress. Int J Pharma BioSciences 1 (2010), 1-6.

[31] J.I. Mäkinen, A. Perheentupa, K. Irjala, P. Pöllänen, J. Mäkinen, I. Huhtaniemi I, Endogenous testosterone and serum lipids in middle-aged men. Atherosclerosis 197(2008), 68893.

[32] G. Millio, E. Corrado, D. Sorrentino, Asymptomatic carotid lesions and aging: role of hypertension and other traditional and emerging risk factors. Arch Med Res 37 (2006), 342-7.

[33] G.F. Fletcher, V. Bufalino, F. Costa, Efficacy of drug therapy in the secondary prevention of cardiovascular disease and stroke. Am J Cardio 99 (2007), 1-35.

[34] P. Libby, Braunwald Tratado de Doenças Cardiovasculares. 7th ed. Elservier Editora Ltd; Biologia Vascular da Aterosclerose (2006), 921-7.

[35] C. Bonithon-Kopp, P.J. Touboul, C. Berr, Relation of intimamedia thickness to atherosclerosis plaques in carotid arteries. The Vascular Aging (EVA) Study. Artheriocler Thromb Vasc Biol 16 (1996), 310-16.

[36] D.H. O'Leary, J.F. Polak, R.A. Kronmal, Thickening of the carotid wall: A marker for atherosclerosis in the elderly? Cardiovascular Health Study Collaborative Research Group. Stroke 27 (1996), 224-31. 


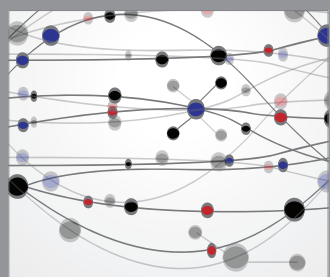

The Scientific World Journal
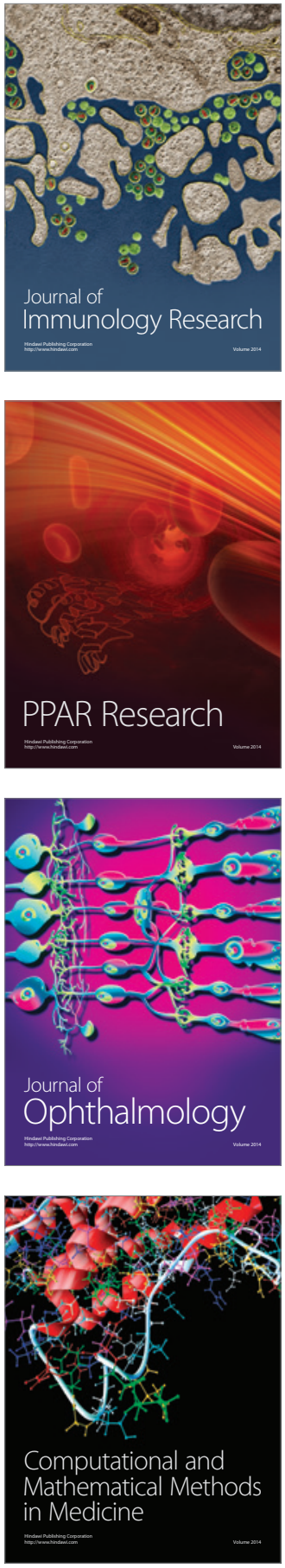

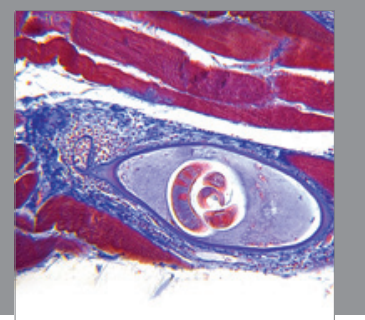

Gastroenterology

Research and Practice
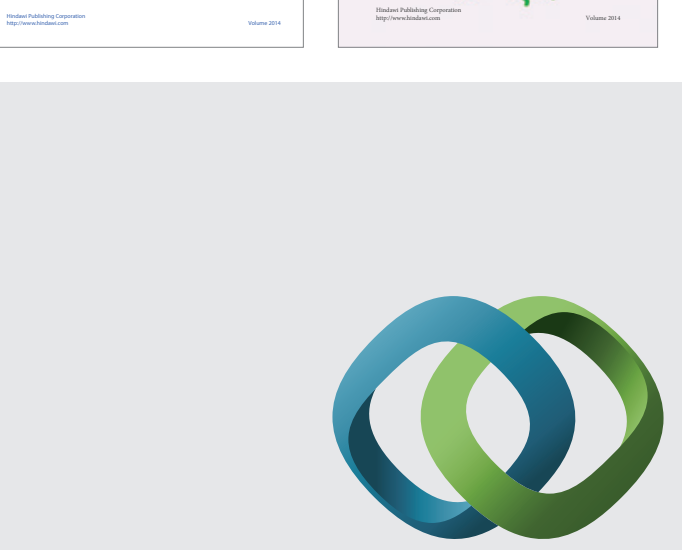

\section{Hindawi}

Submit your manuscripts at

http://www.hindawi.com
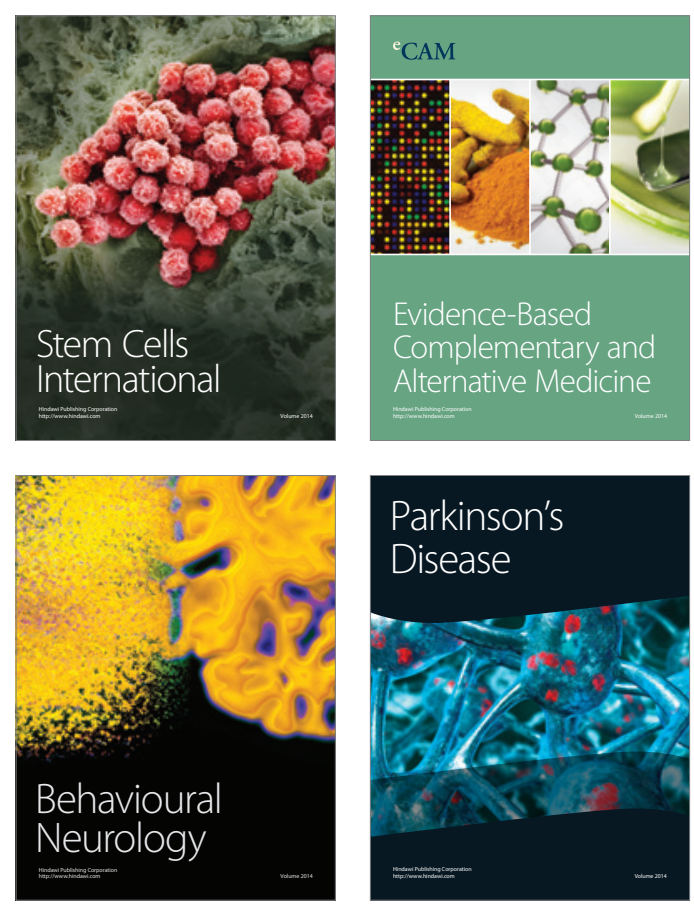

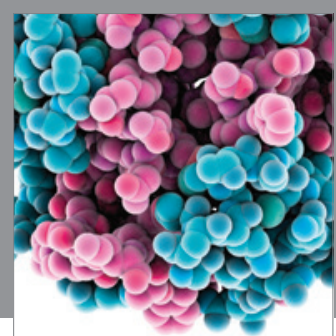

Journal of
Diabetes Research

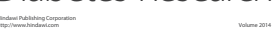

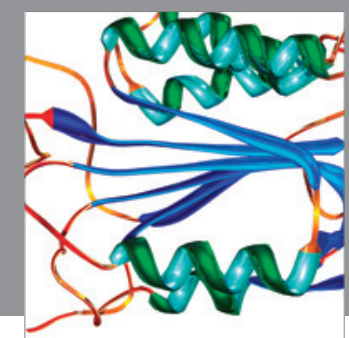

Disease Markers
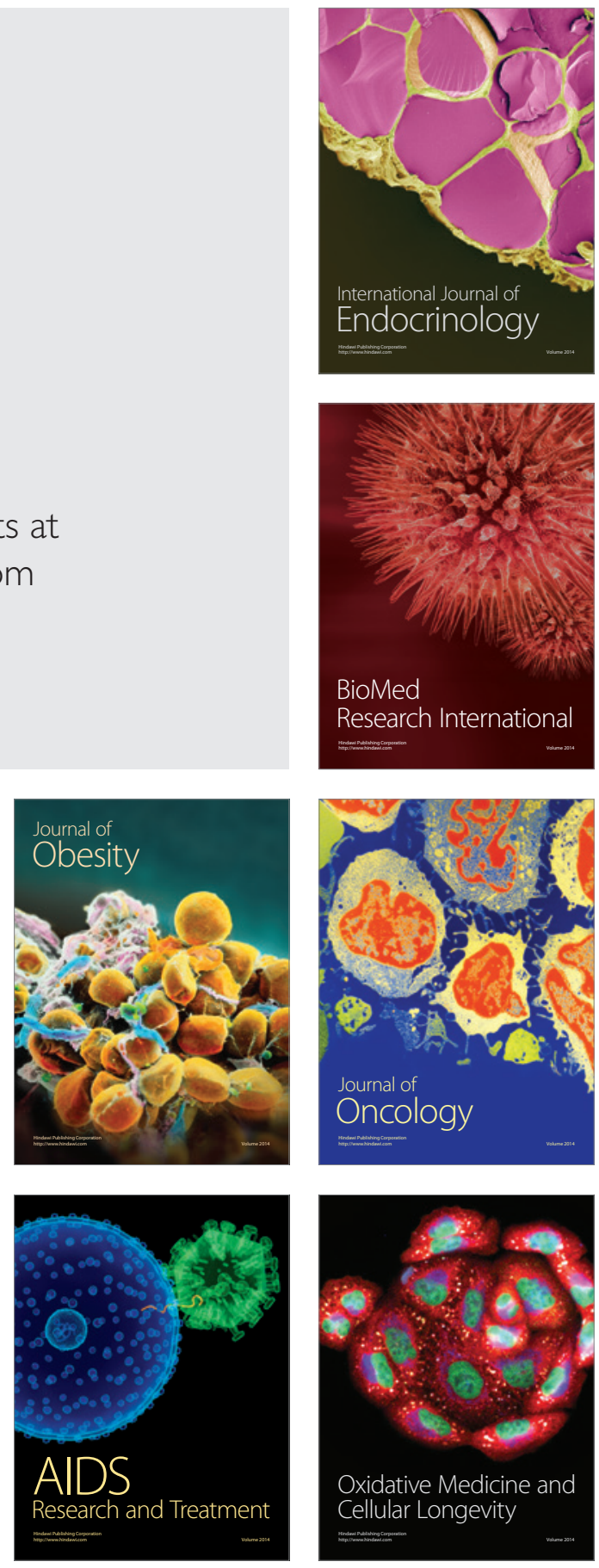